\title{
Spin Dynamics in the Ferromagnetic Resonance
}

\author{
Hongyu Wen, Jianbai Xia* \\ Institute of Semiconductors, University of Chinese Academy of Sciences, Beijing, China
}

Email address:

wenhongyu@semi.ac.cn (Hongyu Wen), xiajb@semi.ac.cn (Jianbai Xia)

${ }^{*}$ Corresponding author:

\section{To cite this article:}

Hongyu Wen, Jianbai Xia. Spin Dynamics in the Ferromagnetic Resonance. American Journal of Physics and Applications.

Vol. 7, No. 1, 2019, pp. 8-13. doi: 10.11648/j.ajpa.20190701.12

Received: December 24, 2018; Accepted: January 21, 2019; Published: February 15, 2019

\begin{abstract}
The LLG equation including the spin-transfer torque term, and the frequency spectrum analysis method are used to study the dynamic process of ferromagnetic resonance. The effects of damping factor $\alpha$, internal anisotropic field, magnetic field inclination, and spin-transfer torque caused by the spin current are studied. The following results are found as follows. The ferromagnetic resonance spectra as functions of the frequency $\omega$ for fixed magnetic field, and functions of magnetic field for fixed frequency are obtained, and it is found that the internal magnetic field also has contribution to the resonance field or frequency, and we know that the resonant frequency $\omega_{0} \approx h_{e}+h_{1}$ (in unit of $\gamma H_{0}$ ). In addition, when the damping factor increases from 0.01 to 0.03 , the resonance frequencies increases slightly, and the resonance strength decreases. And the oscillatory waves of $m_{x}$ and $m_{y}$ reach their stable values more quickly. Furthermore, the internal field perpendicular to the external field $h_{0}$ as well as it parallel to $h_{0}$ also has the effect to the resonant frequency. The positive and negative internal field will have reversed effects to the resonance field or frequency. And in the end when the spin current becomes larger the STT effect becomes stronger, even exceeds the ferromagnetic resonance effect, makes $m_{z}$ reversed, and $m_{x}$ and $m_{y}$ decreased.
\end{abstract}

Keywords: Ferromagnetic Resonance, Frequency Spectrum, Spin-transfer Torque, Internal Anisotropic Field

\section{Introduction}

Ferromagnetic resonance has been used to study the magnetic properties and magnetization dynamics of ferromagnetic material [1-10], such as the Landé $g$ factor, the Gilbert damping parameter $\alpha$, and magnetic anisotropic properties, etc.

Beaujour et al. studied the ferromagnetic resonance of the $\mathrm{Fe}_{1-\mathrm{x}} \mathrm{V}_{\mathrm{x}}$ alloy thin films [11]. The Landé $g$ factor, Gilbert damping parameter $\alpha$ are obtained as functions of the $\mathrm{V}$ component $x$. When $x$ increases the Landé $g$ factor increases from 2.11 for $x=0$ to 2.17 for $x=0.6$. And the damping parameter $\alpha$ also increases from 0.008 for $x=0$ to 0.015 for $x=0.5$. The films exhibit an out-of-plane anisotropy, and the anisotropy constant $K_{\perp}$ decreases with $x$ increasing, from 3.4 $\mathrm{erg} / \mathrm{cm}^{2}$ for $x=0$ decreases to $0.8 \mathrm{erg} / \mathrm{cm}^{2}$ for $x=0.66$. Wu et al. studied the ferromagnetic resonance in a $\mathrm{CoFe} / \mathrm{PtMn} / \mathrm{CoFe}$ multilayer film [12]. In experiments the sample plane was rotated with respect to the direction of the magnetic field. The $g$ factor and the effective magnetic anisotropy parameters of the magnetic film were obtained from the angle dependence of the resonance peaks as: $g=2.01,2 K_{A} / M \sim 0.1 \quad \mathrm{~T}$, $4 \pi M-2 K_{U} / M \sim 1.9$ T. Kakazei et al studied the ferromagnetic resonance of ultrathin $\mathrm{Co} / \mathrm{Ag}$ superlattices on $\mathrm{Si}$ (111) [13]. FMR spectra have been recorded at various polar angles between the sample plane and the magnetic field. From the angle dependence of the resonance field the fitting parameters are obtained: $g=2.07$, the anisotropic field $H_{\text {eff }}=7.83 \mathrm{kOe}$ for $5 \times[$ Co $(4 \AA) / \mathrm{Ag}(4.5 \AA)]$ SL sample. Urban et al. studied the Gilbert damping in single and multilayer ultrathin Fe films: role of interface in nonlocal spin dynamics [14]. They found that the FMR linewidth for the Fe films in the double layer structures was larger than the FMR linewidth in the single Fe films having the same thickness. The additional FMR linewidth scaled inversely with the film thickness, and increases linearly with increasing microwave frequency. These results demonstrate that a transfer of electron angular momentum between the magnetic layers leads to additional relaxation torques. 


\section{LLG Equation}

The LLG equation has been successfully applied to study the spin reversion by the current driven spin torque. In that case the energy and the absolute value of the spin angular momentum are conserved. In the case of ferromagnetic resonance the energy is not conserved, especially at resonance the magnetic moment increases rapidly. In this paper we will use the LLG equation to study the ferromagnetic resonance. The LLG equation is written in Eq. (1), where $m$ is the unit vector of the macro magnetic moment, thus $m^{2}=1$. In studying ferromagnetic resonance we assume that before applying alternating microwave field $m^{2}=1$, after applying alternating microwave field $m^{2} \neq 1$.

The LLG equation can be written as.

$$
\frac{1}{\gamma_{0}} \frac{d m}{d t}=-m \times H-\alpha m \times(m \times H)+a_{J} m \times\left(m \times n_{s}\right)-\alpha a_{J}\left(m \times n_{s}\right)
$$

Where

$$
\gamma_{0}=\frac{\gamma}{1+\alpha^{2}}
$$

$\gamma \approx 2 \mu_{B} / \hbar$ is the gyromagnetic ratio, $\alpha$ is the Gilbert damping constant, $H$ is the total magnetic field, including external magnetic field $H_{e}$ and internal local anisotropic magnetic field $H_{e f f}, n_{s}$ is the unit vector of the magnetization in the fixed layer. $a_{J}$ is a torque constant relative to the spin-polarized current.

$$
\begin{aligned}
& a_{J}=\left(\frac{\hbar}{2 e}\right) \eta\left(\frac{I}{\mu_{0} S d M_{S}}\right) \\
& \left(1+\alpha^{2}\right) \frac{d m}{d \tau}=-m \times h-\alpha m \times(m \times h)-a_{J H} m \times\left(m \times n_{s}\right)+\alpha a_{J H}\left(m \times n_{s}\right)
\end{aligned}
$$

where $\eta$ is the spin polarization of electrons, $I$ is the current, $M_{s}$ is the saturated magnetization, $S$ and $d$ are the area and width of the free layer, respectively. Temporally we don't consider the term related to current $a_{J}$.

In order to transfer Eq. (1) into the dimensionless form, we take the unit of the magnetic field as $H_{0}, H=h H_{0}$. The time unit is taken as $\tau_{0}=1 / \gamma H_{0}, t=\tau \tau_{0}$. In this paper we took $H_{0}=10^{4}$ $\mathrm{A} / \mathrm{m} \sim 1.257 \times 10^{-2} \mathrm{~T}, \not H_{0}=176 \mathrm{GHz} / \mathrm{T} \times 1.257 \times 10^{-2} \mathrm{~T}=2.21 \mathrm{GHz}$. $\tau_{0}=0.45$ ns. With $\tau$ Eq. (1) becomes.
Where $a_{J H}=a_{J} / H_{0}$.

$H$ represents the sum of the internal and external fields on the magnet. The internal magnetic field is responsible for keeping the magnetization pointing along the easy axis. For example, a thin-film magnet oriented in the $x-y$ plane with easy axis along $z$-axis is characterized by.

$$
H_{\text {eff }}=H_{z} m_{z} \hat{z}+H_{x} m_{x} \hat{x}
$$

Representing the internal "uniaxial anisotropy" effective field. It is noticed that the internal field is dependent on the magnetic moment $m$.

$$
\begin{aligned}
& \left(1+\alpha^{2}\right) \frac{d m_{x}}{d \tau}=-m_{y} m_{z} h_{1}-\alpha\left(m_{x}^{3} h_{2}-m^{2} m_{x} h_{2}+m_{x} m_{z}^{2} h_{1}\right)-m_{y} h_{0}+m_{z} h_{3 y}-\alpha\left[\left(m \cdot h_{e}\right) m_{x}-m^{2} h_{3 x}\right] \\
& \left(1+\alpha^{2}\right) \frac{d m_{y}}{d \tau}=m_{x} m_{z} h_{1}-m_{x} m_{z} h_{2}-\alpha\left(m_{y} m_{x}^{2} h_{2}+m_{y} m_{z}^{2} h_{1}\right)-m_{z} h_{3 x}+m_{x} h_{0}-\alpha\left[\left(m \cdot h_{e}\right) m_{y}-m^{2} h_{3 y}\right] \\
& \left(1+\alpha^{2}\right) \frac{d m_{z}}{d \tau}=m_{x} m_{y} h_{2}-\alpha\left(m_{z} m_{x}^{2} h_{2}+m_{z}^{3} h_{1}-m_{z} m^{2} h_{1}\right)-m_{x} h_{0}+m_{z} h_{3 x}-\alpha\left[\left(m \cdot h_{e}\right) m_{z}-m^{2} h_{0}\right]
\end{aligned}
$$

Where

$$
m \cdot h_{e}=m_{x} h_{3 x}+m_{y} h_{3 y}+m_{z} h_{0}
$$

The detail of the calculation see $[15,16]$.

\section{Ferromagnetic Resonance Properties}

\subsection{Variation of Magnetic Moments at Different Alternating Field Frequencies}

Figure 1-3 show the variation of the magnetic moment components with time at $\omega=1.06,1.07,1.08$, respectively. In the calculation we take the dimensionless quantities: $\alpha=0.02$, $h_{0}=1, h_{1}=0.1, h_{2}=0, h_{3}=0.02$. The initial conditions are: $m_{x}=0.01, m_{y}=0, m_{z}=1$. From Figure $1-3$ we see that the magnetic moment components oscillate with the alternation field frequency, the period is $\tau_{0}=2 \pi / \omega$, but the amplitudes are different. At a definite frequency $\omega_{0}$ the resonance occurs, the amplitude is largest, the magnetic moment components $m_{x}$ and $m_{y}$ increase rapidly with time, that is ferromagnetic resonance. At the same time, the $m_{z}$ decreases with time, it decreases largest at resonance. Because except the external constant field $h_{0}=1$, there is also internal anisotropic field $h_{1}=0.1$ in the $z$ direction, thus the resonant frequency is approximately determined by the sum of the two magnetic fields, though the properties of the two fields are different, the letter is $m_{z}$ 
dependent. The resonant frequency $\omega_{0} \approx h_{0}+h_{1}=1.1$ (in unit of $\left.\gamma_{0}\right)$.

From Figure 1 we can see that the amplitudes of $m_{x}$ and $m_{y}$
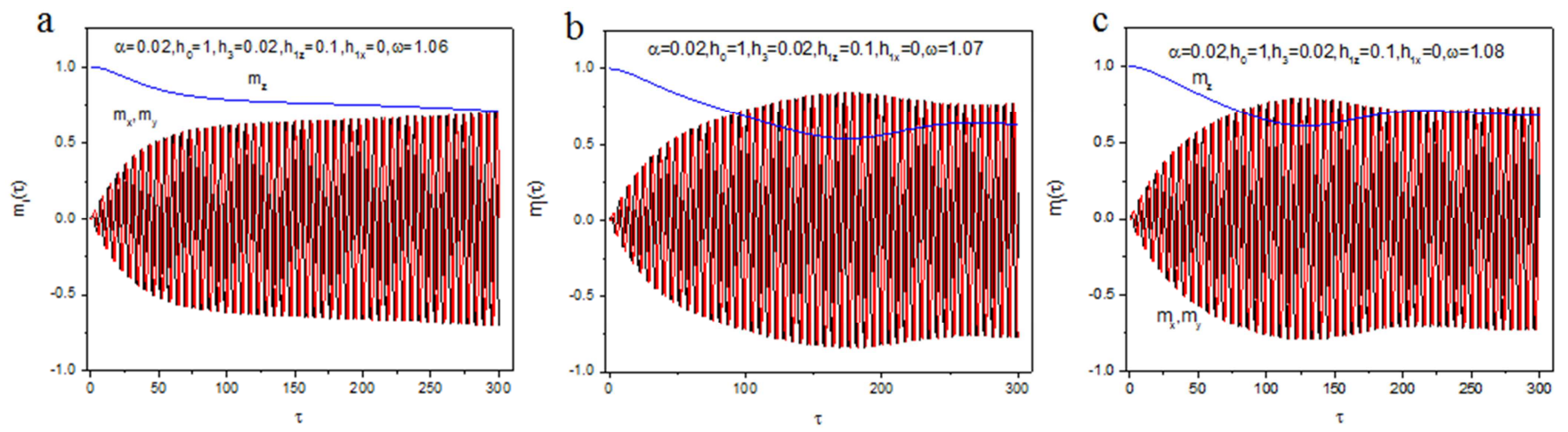

Figure 1. Variation of the magnetic moment components with time for $h_{0}=1, h_{1}=0.1, h_{2}=0, h_{3}=0.02, \alpha=0.02$. (a) $\omega=1.06$. (b) $\omega=1.07$. (c) $\omega=1.08$, respectively.

\subsection{Ferromagnetic Resonance Spectrum}

We should transfer the ferromagnetic oscillations $m_{i}(t)(i=x$, $y$ ) in the last section Figure 1, into the frequency spectrum $m_{i}$ $\omega)$, i.e. the ferromagnetic resonance spectra. By use of the sine Fourier transform for the $m_{x}$ oscillation,

$$
F(\omega)=\lim _{a \rightarrow \infty} \frac{4}{\pi a}\left[\int_{0}^{a} f(t) \sin \omega t d t\right]^{2}
$$

Where $a$ is a large number respective to the oscillating period. For example, $f(t)=\sin \omega_{0} t$, then.

$$
\int_{0}^{a} \sin \omega_{0} t \sin \omega t d t=\frac{1}{2}\left[\cos \left(\omega_{0}-\omega\right) t-\cos \left(\omega_{0}+\omega\right) t\right]=\frac{1}{2}\left[\frac{\sin \left(\omega_{0}-\omega\right) a}{\left(\omega_{0}-\omega\right)}-\frac{\sin \left(\omega_{0}+\omega\right) a}{\left(\omega_{0}+\omega\right)}\right]
$$

From the definition of the $\delta$ function,

$$
\delta(x)=\lim _{a \rightarrow \infty} \frac{1}{\pi} \frac{\sin ^{2} a x}{a x^{2}}
$$

We obtain,

$$
F(\omega)=\lim _{a \rightarrow \infty} \frac{4}{\pi a} \frac{\sin ^{2}\left(\omega_{0}-\omega\right) a}{4\left(\omega_{0}-\omega\right)^{2}}=\delta\left(\omega_{0}-\omega\right)
$$

Similarly we use the cosine Fourier transform for the $m_{y}$ oscillation.

Because the $m_{i}(\tau)$ is given by the numerical method at the discrete points of dimensionless time points $\tau_{\mathrm{n}}$, we integrate the equation (9) by the fixed step Simpson integrating method. Figure 2 is the frequency spectrum for $h_{0}=1, h_{1}=0.1, h_{2}=0$, $h_{3}=0.02, \alpha=0.02$, at $\omega=1.096$ (resonance frequency), where the units of time and frequency are dimensionless $\tau$ and $2 \pi / \tau$.

In figure 2, there are two curves of $m_{x}$ and $m_{y}$, they are not distinguished as desired. From Figure 2 we see that there are three main resonance peaks for $m_{x}$ and $m_{y}$, respectively. The position of the main peak is around $\omega_{0}=1.096$. There are also two other harmonic waves for each component, i.e. ferromagnetic resonance waves, but the frequencies are different from the main frequency, and the amplitudes are smaller.

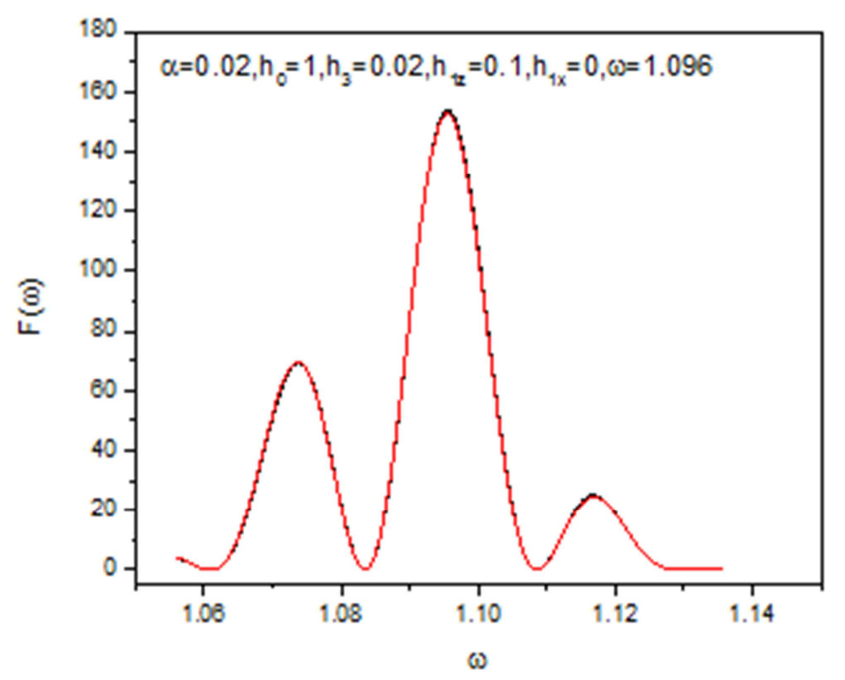

Figure 2. Frequency spectra of $m_{x}$ and $m_{y}$ at resonance frequency $\omega_{0}=1.096$.

\subsection{Ferromagnetic Resonance Spectra}

Figure 3 are the ferromagnetic resonance spectrum for $h_{1}=0.1, h_{2}=0, h_{3}=0.02, \alpha=0.02$, (a) $h_{0}=1, \omega$ is the variable quantity, (b) $\omega=1, h_{0}$ is the variable quantity, respectively. 

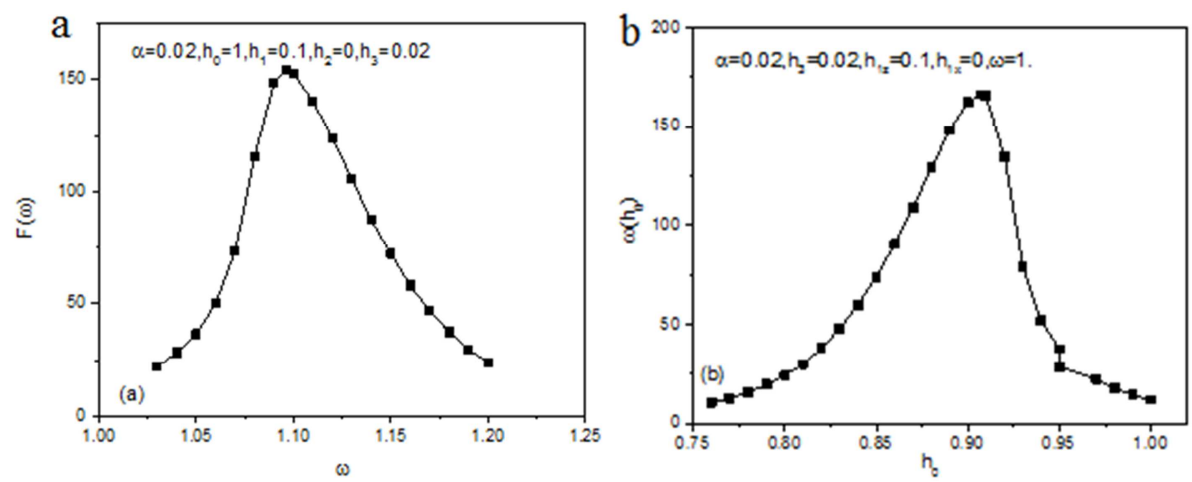

Figure 3. Ferromagnetic resonance spectrum for $h_{1}=0.1, h_{2}=0, h_{3}=0.02, \alpha=0.02$, (a) $h_{0}=1$, $\omega$ is the variable quantity. (b) $\omega=1$, $h_{0}$ is the variable quantity.

Comparing Figure3 (a) and (b) we found that the shape of resonance peak is more symmetric for $\omega$ as a variable, while it is non-symmetric for $h_{0}$ as a variable. In Figure 3 (a) $h_{0}=1$, $h_{1}=0.1$ in the $z$ direction, the resonance frequency $\omega_{0}=1.096 \approx$ $h_{0}+h_{1}$. In Figure 3 (b) $\omega=1$, the resonance magnetic field $h_{0}=0.907 \approx \omega-h_{1}$. It is noticed that here we use the dimensionless quantities so the values of $\omega$ and $h$ are the same.

\subsection{Effect of Damping Factor $\alpha$}

In Figure 4 the ferromagnetic resonance spectrum for $h_{0}=1$, $h_{1}=0.1, h_{2}=0, h_{3}=0.02$, and $\alpha=0.01,0.02,0.03$ are shown as functions of $\omega$. From Figure 4 we see that the resonance strength is smaller when the damping factor $\alpha$ is larger, and the resonance frequency blue shifts slightly, equal 1.09, 1.096, and 1.11 , respectively.

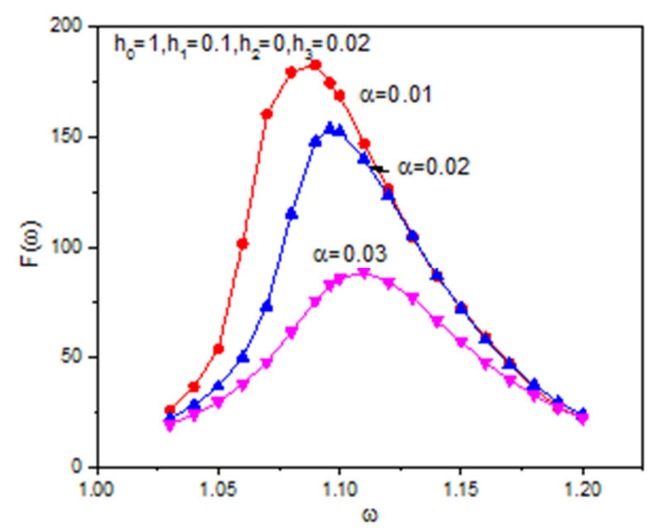

Figure 4. Ferromagnetic resonance spectrum for $h_{0}=1, h_{1}=0.1, h_{2}=0$, $h_{3}=0.02$, and $\alpha=0.01,0.02,0.03$ as functions of $\omega$.

\subsection{Effect of Internal Anisotropic Field $h_{1}$ and $h_{2}$}

In this paper we assume that the external magnetic $h_{0}$ is in the $z$ direction, the internal anisotropic field $h_{1}$ is also in the $z$ direction, while the internal anisotropic field $h_{2}$ is in the $x$ direction, perpendicular to $h_{0}$. Besides, the internal fields are magnetic moment dependent, see Eq. (6).

Figure 5 (a) and (b) shows the frequency spectra and $m_{x}$ and $m_{y}$ for $\alpha=0.02, h_{0}=1, h_{1}=0, h_{3}=0.02, \omega=1$, and $h_{2}= \pm 0.1$, respectively. From Figure 5 we see that due to breaking of the axial symmetry in the $z$ direction the curves of $m_{x}$ and $m_{y}$ are different. For $h_{2}=+0.1 m_{x}>m_{y}$, for $h_{2}=-0.1 m_{x}<m_{y}$.
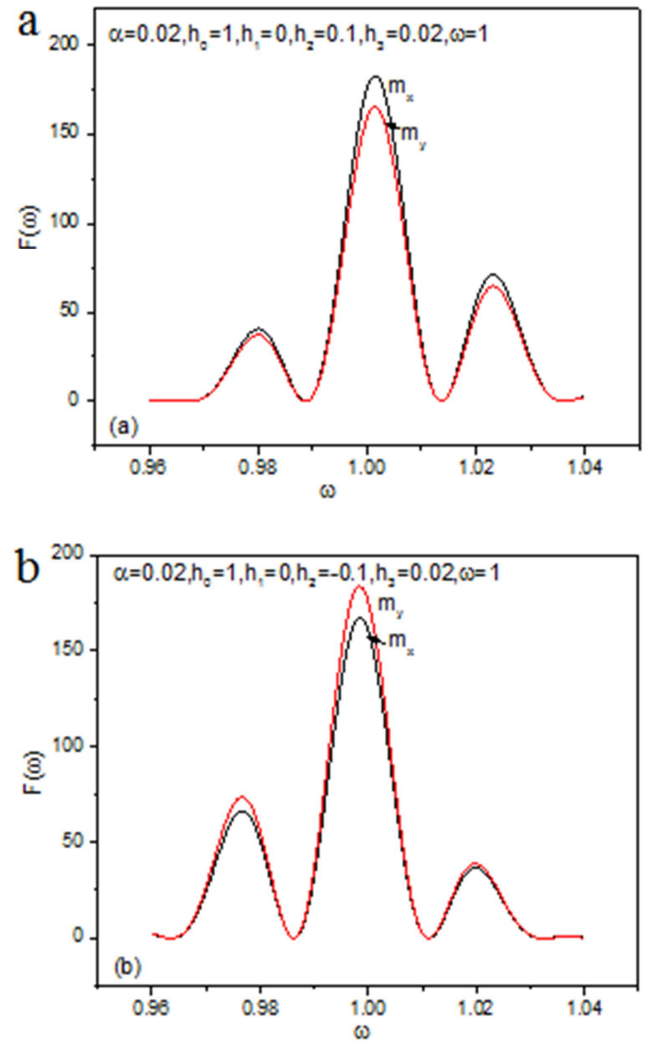

Figure 5. Frequency spectra and $m_{x}$ and $m_{y}$ for $\alpha=0.02, h_{0}=1, h_{1}=0, h_{3}=0.02$, $\omega=1$, and (a) $h_{2}=+0.1$ (b) $h_{2}=-0.1$.

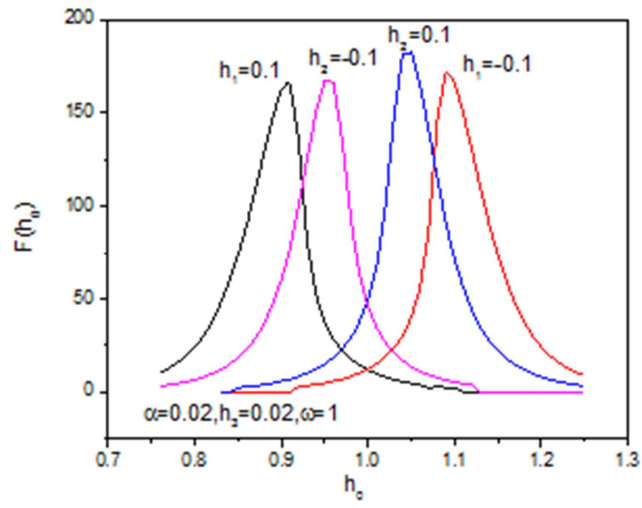

Figure 6. Ferromagnetic resonance spectra as functions of $h_{0}$ for $\alpha=0.02$, $h_{3}=0.02, \omega=1$, and $h_{1}= \pm 0.1, h_{2}=0$, or $h_{1}=0, h_{2}= \pm 0.1$, respectively. 
Figure 6 shows the ferromagnetic resonance spectra (from $m_{x}$ ) as functions of $h_{0}$ for $\alpha=0.02, h_{3}=0.02, \omega=1$, and $h_{1}= \pm 0.1$, $h_{2}=0$, or $h_{1}=0, h_{2}= \pm 0.1$, respectively. As shown in Sec. 3.3 because the internal field $h_{1}$ is in the $z$ direction, it will influence the resonance fields, which are 0.907 and 1.09 for $h_{1}=+0.1$ and $h_{1}=-0.1$, respectively. While the internal field $h_{2}$ is in the $x$ direction, it also influence the resonance fields, which are 0.95 and 1.05 for $h_{2}=-0.1$ and $h_{2}=0.1$, respectively. When the absolute value of the internal field increases the resonance field will extend to both sides as in Figure 6.

\subsection{Effect of Magnetic Field Inclination}

In the previous calculations we assumed that the external magnetic field $H_{0}$ is always in the $\mathrm{z}$ direction, here we consider the effect of the magnetic field inclination. Assume that the inclination angle between $H_{0}$ and the $z$ axis is $\theta$, then the external magnetic field

$$
H_{0 z}=H_{0} \cos \theta, \quad H_{0 x}=H_{0} \sin \theta .
$$

Figure 7 shows the frequency spectrum of $m_{x}$ and $m_{y}$ for $\theta=15^{\circ}, \quad \alpha=0.02, \quad h_{0}=1, \quad h_{1}=0.1, \quad h_{2}=0, \quad h_{3}=0.02, \quad \omega=1.09$. Comparing with Figure2, where $\theta=0^{\circ}$, the curves of $m_{x}$ and $m_{y}$ are not duplicated again due to the axial symmetry breaking.

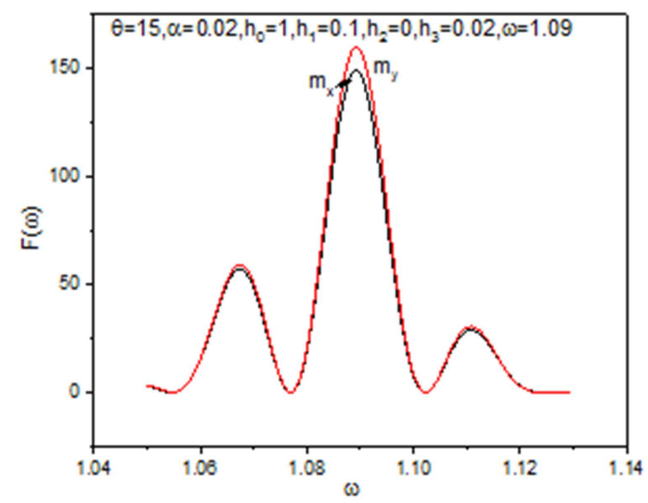

Figure 7. Frequency spectrum of $m_{x}$ and $m_{y}$ for $\theta=15^{\circ}, \alpha=0.02, h_{0}=1, h_{1}=0.1$, $h_{2}=0, h_{3}=0.02, \omega=1.09$.

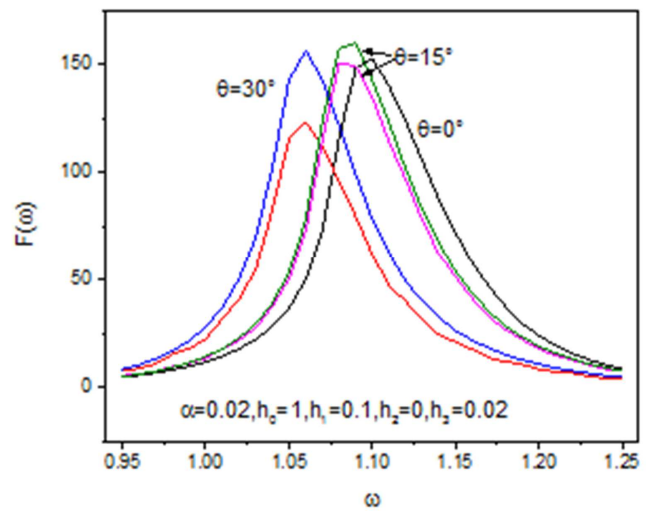

Figure 8. Ferromagnetic resonance spectra as functions of $\omega$ for $\alpha=0.02$, $h_{0}=1, h_{1}=0.1, h_{2}=0, h_{3}=0.02$, and $\theta=0^{\circ}, 15^{\circ}, 30^{\circ}$.

Figure 8 shows the ferromagnetic resonance spectra as functions of $\omega$ for $\alpha=0.02, h_{0}=1, h_{1}=0.1, h_{2}=0, h_{3}=0.02$, and $\theta=0^{\circ}, 15^{\circ}, 30^{\circ}$, respectively. In the figure there are two curves for $\theta=15^{\circ}$ and $30^{\circ}$, corresponding to $m_{y}$ and $m_{x}$, respectively $\left(m_{y}>m_{x}\right)$. The resonance frequency $\omega_{0} \sim \cos \theta$ decreases as the $\theta$ increases.

\subsection{Effect of Spin-Transfer Torque (SST) Caused by Spin Current}

We suppose that in the case of ferromagnetic resonance a spin current $J$ is applied to the sample from the fixed ferromagnetic layer to the free ferromagnetic layer, which is represented by the $a_{J}$ term in the Eq. (1). Now consider the effect of the STT caused by spin current. When the current value $a_{J}$ is smaller than a critical value the magnetic moment $m_{z}$ will not reverse, when the electric current $a_{J}$ exceeds the critical value the $m_{z}$ will reverse [5], this is the principle of the SST. We study the ferromagnetic resonance in this process.

Figure 9 shows the frequency spectra of $m_{x}$ and $m_{y}$ for $\alpha=0.02, h_{0}=0.907, h_{1}=0.1, h_{2}=0, h_{3}=0.02, \omega=1$, and $J_{H}=0$, $0.02,0.04$, respectively. Where $J_{H}$ is the dimensionless current parameter, see [15]. When $J_{H}=0.02$, the $m_{z}$ does not reverse, when $J_{H}=0.04$, the $m_{z}$ reverses. From Fig. 9 we see that along with the current increases there will be more resonance peaks, the peak frequency and height decrease. The STT effect will exceeds the ferromagnetic resonance effect.

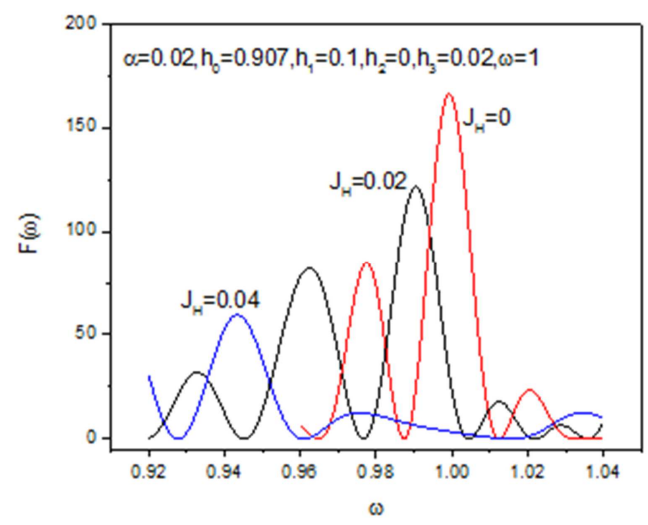

Figure 9. Frequency spectra of $m_{x}$ and $m_{y}$ for $\alpha=0.02, h_{0}=0.907, h_{1}=0.1, h_{2}=0$ $h_{3}=0.02, \omega=1$., and $J_{H}=0,0.02,0.04$.

\section{Summary}

We use the LLG equation including the spin-transfer torque term, and without the constraint of magnetic moment conservation to study the dynamic process of ferromagnetic resonance. We studied the effects of damping factor $\alpha$, internal anisotropic field, magnetic field inclination, and spin-transfer torque caused by the spin current, and obtained the following results:

1. We obtained the ferromagnetic resonance spectra as functions of the frequency $\omega$ for fixed magnetic field, and functions of magnetic field for fixed frequency from the solutions of LLG equation. It is found that the internal magnetic field also has contribution to the resonance field or 
frequency. The resonant frequency (or field) is approximately determined by the sum of the two magnetic fields, though the properties of the two fields are different, the letter is $m_{z}$ dependent. The resonant frequency $\omega_{0} \approx h_{e}+h_{1}$ (in unit of $\gamma H_{0}$ ).

2. When the damping factor increases from 0.01 to 0.03 , the resonance frequencies increases slightly, and the resonance strength decreases. The oscillatory waves of $m_{x}$ and $m_{y}$ reach their stable values more quickly.

3. The internal field perpendicular to the external field $h_{0}$ as well as it parallel to $h_{0}$ also has the effect to the resonant frequency. The resonant fields are $h_{0}=0.907\left(h_{1}=0.1, h_{2}=0\right)$, $0.95\left(h_{1}=0, h_{2}=-0.1\right), 1.05\left(h_{1}=0, h_{2}=0.1\right), 1.09\left(h_{1}=-0.1, h_{2}=0\right)$ for $\omega=1 ., h_{3}=0.02$, respectively. The positive and negative internal field will have reversed effects to the resonance field or frequency.

4. When the spin current becomes larger the STT effect becomes stronger, even exceeds the ferromagnetic resonance effect, makes $m_{z}$ reversed, and $m_{x}$ and $m_{y}$ decreased.

\section{Acknowledgements}

This work was supported by the Advanced Research Plan of Chinese Academy of Sciences: QYZDY-SSW-JSC015.

\section{References}

[1] Charles Kittel, On the Theory of Ferromagnetic Resonance Absorption. Phys. Rev. 73, 155 - Published 15 January 1948.

[2] Michael Farle, Ferromagnetic resonance of ultrathin metallic layers. Rep. Prog. Phys. 61 (1998) 755-826.

[3] Luqiao Liu, Takahiro Moriyama, D. C. Ralph, and R. A. Buhrman, Spin-Torque Ferromagnetic Resonance Induced by the Spin Hall Effect. Phys. Rev. Lett. 106, 036601.

[4] L. R. Walker, Magnetostatic Modes in Ferromagnetic Resonance, Phys. Rev. 105, 390- Published 15 January 1957.

[5] Nick S. Norberg†, Kevin R. Kittilstved†, Synthesis of
Colloidal $\mathrm{Mn}^{2+}: \mathrm{ZnO}$ Quantum Dots and High-TC Ferromagnetic Nanocrystalline Thin Films. J. Am. Chem. Soc., 126 (30), pp 9387-9398 (2004).

[6] Rodrigo Arias and D. L. Mills, Extrinsic contributions to the ferromagnetic resonance response of ultrathin films, Phys. Rev. B 60,7395.

[7] N. Bloembergen and S. Wang, Relaxation Effects in Para- and Ferromagnetic Resonance, Phys. Rev. 93, 72

[8] Arne Brataas, Yaroslav Tserkovnyak, Spin battery operated by ferromagnetic resonance, Phys. Rev. B 66, 060404

[9] Sangita S. Kalarickal, Pavol Krivosik, Ferromagnetic resonance linewidth in metallic thin films: Comparison of measurement methods, Journal of Applied Physics 99, 093909 (2006).

[10] J. C. Sankey, P. M. Braganca, A. G. F. Garcia, Spin-Transfer-Driven Ferromagnetic Resonance of Individual Nanomagnets, Phys. Rev. Lett. 96, 227601 (2006).

[11] Beaujour, J. M. L., Kent, A. D., Abraham, D. W., Sun, J. Z., Ferromagnetic resonance study of polycrystalline $\mathrm{Fe}_{1-\mathrm{x}} \mathrm{V}_{\mathrm{x}}$ alloy thin films. Journal of Applied Physics 103, 07B519 (2008).

[12] Wu C, Khalfan A N, Pettiford C, Ferromagnetic resonance studies of surface and bulk spin-wave modes in a $\mathrm{CoFe} / \mathrm{PtMn} / \mathrm{CoFe}$ multilayer film. Appl. Phys. 103, 07B525 (2008).

[13] Kakazei G N, Martin P P, Ruiz A, et al. Ferromagnetic resonance of ultrathin $\mathrm{Co} / \mathrm{Ag}$ superlattices on $\mathrm{Si}$ (111) J. Appl. Phys. 103, 07B527 (2008)

[14] Ulban R, Woltersdorf G, Heinrich B, Gilbert Damping in Single and Multilayer Ultrathin Films: Role of Interfaces in Nonlocal Spin Dynamics Phys. Rev. Lett. 87, 217204 (2001).

[15] Wen, H. Y, Xia, J. B, Control of spins in a nano-sized magnet using electric-current. Chin. Phys. B. 26, 047501 (2017).

[16] Wen, H. Y, Xia, J. B, Voltage control of magnetization switching and dynamics. Chin. Phys. B. 27, 067502 (2018). 